\title{
Fungal pathogens and primary antifungal prophylaxis in patients with hematological malignancies: one year experience
}

\author{
Gedik $\mathrm{H}^{1}$, Yildirmak $\mathrm{MT}^{1}$, Simsek $\mathrm{F}^{1}$, Aydin $\mathrm{D}^{2}$, Demirel $\mathrm{N}^{2}$, Yokus $\mathrm{O}^{2}$, Arica $\mathrm{D}^{2}$
}

1. Department of Infectious diseases and Clinical Microbiology, Ministry of Health Okmeydani Training and Research Hospital, Istanbul

2. Department of Hematology, Ministry of Health Okmeydani Training and Research Hospital, Istanbul

\begin{abstract}
Background: Febrile neutropenia (FN) is generally a complication of cancer chemotherapy in patients with hematological malignancies.

Objective: To evaluate the febrile neutropenia episodes of hematological patients and their outcomes with respect to fungal pathogens, primary antifungal prophylaxis antifungal therapy.

Methods: All consecutive patients older than 14 years of age and who developed febrile neutropenia episodes from September 2010 to November 2011 were incorporated into this study.

Results: In total, we retrospectively evaluated 86 consecutive patients and their 148 neutropenic episodes. Of the 86 patients, 45 were male and the mean age was 47,65 $\pm 15,06$ years (range: $17-82$ years). The mean MASCC score was $18,72 \pm$ 9,43 . Systemic antifungal drug was initiated to 17 patients with probable fungal infection and 12 patients with possible fungal infection. Of seven patients who received posaconazole prophylaxis, five were treated with systemic fungal infection due to possible fungal infection.

Conclusions: It is obvious that more studies focused on primary prophylaxis are needed and primary or secondary antifungal prophylaxis should be evaluated in terms of provided benefits and disadvantages. Timely and appropriately initiated antifungal treatment is one of the most important factors for a good prognosis for recovery from a neutropenic phase.
\end{abstract}

Key words: Hematological patients, febrile neutropenia, antifungal prophylaxis, antifungal treatment African Health Sciences 2012; 12 (3): 390 - 394 http://dx.doi.org/10.4314/ahs.v12i3.23

\section{Introduction}

Febrile neutropenia $(\mathrm{FN})$ is generally a complication of cancer chemotherapy ${ }^{1}$. Mortality related with FN has steadily decreased with guidelines, new laboratory tests and serial computed tomography (CT) scanning, with overall mortality rates ranging from as low as $5 \%$ in patients with solid tumors $1 \%$ in low-risk patients) and as high as $11 \%$ in some hematological malignancies $^{2}$. Multinational Association of Supportive Care in Cancer (MASCC) prognostic

\footnotetext{
*Correspondence author:

Habip Gedik

Department of Infectious diseases and Clinical Microbiology

Ministry of Health Okmeydani Training and

Research Hospital

Istanbul, Turkey

Phone: 009050533627 70, +902123145555

E-mail: habipgedik@yahoo.com
}

index indicates that mortality is as low as 3\% if the MASCC score is $>21$, but as high as $36 \%$ if the MASCC score is $<15^{3}$. Invasive fungal infections associated with aspergillosis, disseminated candidiasis, Fusarium, Zygomycetes, etc., are diminished among patients who have prolonged neutropenia and higher MASCC scores, and/or persistent or prolonged fever after 3-7 days of appropriate treatment ${ }^{2}$. Additionally, patients who have acute myeloid leukemia during remission induction chemotherapy and undergo allogeneic hematopoietic stem cell transplantation with prior conditioning chemotherapy are at risk of invasive aspergillosis due to prolonged and profound neutropenia ${ }^{2}$. Empirical antifungal therapy is recommended for presenting persistent neutropenic fever despite 4-7 days of empirical antibiotic therapy ${ }^{4}$. However, invasive fungal infection was demonstrated among only $4 \%$ of patients who comprised $22 \%-34 \%$ of the 
neutropenic patients who had cancer and received an antifungal drug according to established criteria ${ }^{5}$. CT scanning and galactomannan, with a reported sensitivity rate of $58 \%-65 \%$ and specificity of $65 \%$ $95 \%$ are recommended for preemptive antifungal therapy ${ }^{1}$.

Here, we retrospectively evaluated the febrile neutropenia episodes of hematological patients and their outcomes with respect to fungal pathogens, primary antifungal prophylaxis antifungal therapy.

\section{Methods}

All consecutive patients between September 2010 and November 2011, who were older than 14 years of age and who developed febrile neutropenia episodes during chemotherapy due to hematological cancers in the hematology department at the Ministry of Health, Okmeydaný Training and Research Hospital, Istanbul, Turkey were included in the study. The hematology department is equipped with 23 beds. Patients rooms were designed as single, double and four-person without HEPA filters. Febrile neutropenia ( $\mathrm{FN}$ ) was defined as an oral temperature $>38.5^{\circ} \mathrm{C}$ or two consecutive readings of $>38.0^{\circ} \mathrm{C}$ for $2 \mathrm{~h}$ and an absolute neutrophil count $<0.5 \times$ $10^{9} / \mathrm{L}$, or expected count to fall below $0.5 \times 10^{9} /$ $\mathrm{L}^{1}$.

\section{Collected data included patient} demographics and diagnosis, episode data, clinical presentation and laboratory findings, clinical therapy, microbiological data and outcome. The febril neutropenia treatment protocol was based on clinical practice guidelines for the use of antimicrobial agents in neutropenic patients with cancer: from 2002 and the update in 2010 by the Infectious Diseases Society of America ${ }^{1,6}$.

Empirical antifungal treatment was considered for patients with persistent or recurrent fever after 4-7 days of antibiotics and whose overall duration of neutropenia was expected to be more than 7 days. If CT showed changes associated with fungal infection, amphotericin $\mathrm{B}$ (conventional or liposomal, AM-B) or voriconazole (VOR) were initiated. Cavitation, air-crescent sign and halo sign were classified as the major changes. Nodules and new infiltrates, including consolidation and effusions, were classified as minor changes ${ }^{7}$. Caspofungin (CAS) was chosen as the empirical treatment for patients without the above-mentioned pulmonary findings. Galactomannan assay could not be implemented for all patients due to the unavailability of the test in the laboratory.
Primary prophylaxis against invasive Aspergillus infections with posaconazole (POS) was considered for patients who were undergoing intensive chemotherapy for acute myeloid leukemia (AML) or myelodysplastic syndrome (MDS) in whom the risk of invasive aspergillosis without prophylaxis was substantial. Secondary antifungal prophylaxis with either POS or VOR was considered for patients who had proven or probable or possible fungal infection that responded to antifungal treatment under chemotherapy. Antifungal prophylaxis was administered to patients during the neutropenic phase and it was discontinued after recovery from neutropenia. Blood samples drawn from vein or catheter were inoculated into BactAlert 3D bottles (bioMérieux Diagnostics, Lyon, France). In addition, samples from urine, sputum, wound, conjunctive, abscess, blood and catheter were inoculated onto 5\% sheep-blood agar (Salubris Inc., Istanbul, Turkey), or chocolate agar (Salubris Inc., Istanbul, Turkey) and MacConkey agar (Salubris Inc., Istanbul, Turkey). Isolated yeasts from blood cultures were identified by morphologic examination on Sabouraud Dextrose Agar (Unipath Ltd., Basingstoke, England) plates, germ-tube formation and API ID 32C (bioMérieux Diagnostics, Lyon, France) at the species level.

The ATB Fungus 2 microdilution kit was used (bioMérieux Diagnostics, Lyon, France) for susceptibility testing according to the CLSI (formerly NCCLS) broth microdilution M27-A2 protocol. Aspergillus galactomannan antigen test was performed using the Platelia commercial enzyme immunoassay kit (Platelia Aspergillus EIA; BioRad Laboratories, Marnes-la-Coquette, France). Blood samples of patients were analyzed twice weekly and results were computed as an index which 0.5 and greater was considered as positive relative to optical density of the control sample measured with a semiautomatic analyzer (Behring ELISA processor III; Dade Behring, Marburg, Germany) ${ }^{8}$. Possible causes of false-positive and-negative results were eliminated. The test was considered significant if patient had two consecutive galactomannan assays with an index of 0.5 and greater under persistent or recurrent fever after 4-7 days of antibiotics with or without microbiologic or radiological findings associated with fungal infections as mentioned above and whose overall duration of neutropenia was expected to be more than 7 days ${ }^{1}$.

Response to treatment was defined as defervescence in 48-72 hours subsequent to initiation 
antifungal therapy, recovery of increased $\mathrm{C}$ reactive protein (CRP) level, leukocytosis or leukopenia, recovery of vital signs and clinical symptoms associated with infection (improvement in arterial blood-gas values, radiological improvement, negative urine culture for urinary system infection and recovery of signs and symptoms related to other infections). In-hospital mortality during the neutropenic phase and the clinical outcomes of febrile neutropenic episodes were the primary consequences that were investigated in this study.

\section{Statistical analysis}

Continuous variables were described as mean \pm standard deviation and range. Percentile values were described without decimals. Overall mortality associated with febrile neutropenia was defined as death within 30 days after development of neutropenia.

\section{Results}

We retrospectively analyzed 86 consecutive patients with neutropenia and their 148 febrile episodes. Of the 86 patients, 45 were male and the mean age was $47,65 \pm 15,06$ years (range: $17-82$ years). Mean MASCC score was $18,72 \pm 9,43$. Hematological malignancies of the patients are presented in Table 1.

Table.1: Distribution of hematologic malignancies in the patients with febrile neutropenia (n:86)

\begin{tabular}{lr}
\hline Hematologic Malignancies & n (\%) \\
\hline Acute myeloblastic leukemia & $50(58)$ \\
Acute lymphocytic leukemia & $19(22)$ \\
Chronic lymphocytic leukemia & $5(6)$ \\
Multiple myeloma & $4(4)$ \\
Non-Hodgkin lymphoma & $2(3)$ \\
Mantle-cell lymphoma & $2(3)$ \\
Aplastic anemia & $1(1)$ \\
Hairy cell leukemia & $1(1)$ \\
Chronic lymphocytic leukemia with & $1(1)$ \\
Burkitt's lymphoma & \\
Chronic myeloid leukemia & $1(1)$ \\
\hline
\end{tabular}

Systemic antifungal drug was initiated to 17 patients with probable fungal infection and 12 patients with possible fungal infection. There was no proven infection associated with Zygomycetes or Fusarium species. Isolated fungal pathogens are presented in table 2 .

Table.2: Isolated fungal pathogens and antifungal treatment response in the patients with febrile neutropenia

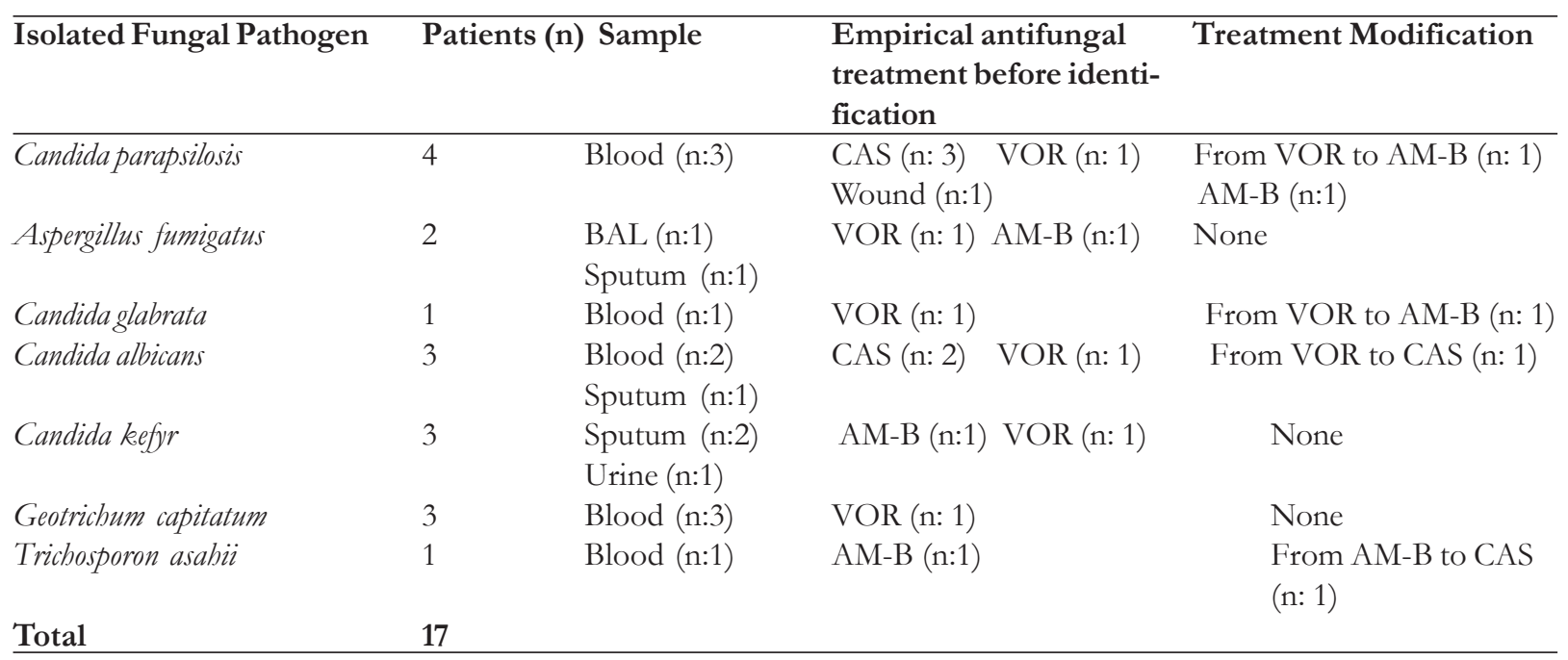

BAL: Bronchoalveolar lavage, VOR: Voriconazole, AM-B: Liposomal Amphotericin B, CAS: Caspofungin 
Voriconazole and fluconazole resistance were identified in each isolate of Candidaparapsilosis, Candida glabrata and Candida albicans species. In total, five patients with acute myeloid leukemia and two patients with acute lymphocytic leukemia were treated with a combination of antifungal drugs due to persistent fever and negative culture and deterioration. Empirical antifungal therapy was initiated with AMB in seven patients, CAS in twelve patients and VOR in ten patients who had thorax CT findings compatible with pulmonary fungal infection. VOR and liposomal AM-B combination were used in two patients.

One of the patients responded to treatment, while the other could not tolerate treatment due to hallucinations, a known side effect of VOR. VOR and CAS combination were used in four patients empirically and none of them responded to treatment. Liposomal AM-B and CAS combination were used in two patients empirically, one of whom responded to treatment. POS prophylaxis was given to seven patients.

One patient with colostomy could not tolerate it due to diarrhea, and POS was modified to an intravenous antifungal drug in four patients due to persistent fever, new infiltration in thorax CT, and deterioration. Only two patients did not need antifungal treatment under induction chemotherapy, whereas five patients who experienced POS primary prophylaxis received systemic antifungal treatment due to possible fungal infection. POS maintained clinical and microbiologic responses that were achieved with either liposomal AM-B or CAS in the patients due to possible and probable fungal infections. Overall mortality rates were 26\% (n:23) and 17 patients died due to infection. MRSA bacteremia (n:2), candidemia (n:5) and vancomycin sensitive Enterococcus faecium that induced severe sepsis (n:1) were recorded in patients who died.

\section{Discussion}

Our patients did not exhibit the expected benefits of primary prophylaxis with POS, although they were a small group. Studies related to POS prophylaxis versus fluconazole prophylaxis in the above-mentioned group of patients revealed a lower incidence of neutropenia in the patient group that received $\operatorname{POS}^{9,10}$. However, when probable cases are removed, the advantage in using POS disappears ${ }^{11}$. In the abstract that Gomes and colleagues presented at the European Congress of Clinical Microbiology and Infectious Diseases (ECCMID) in 2012, antifungal drugs including VOR (intravenous and oral), fluconazole, ecinocandins (CAS, micafungin and andilafungin) were compared for effectiveness in primary antifungal prophylaxis.

Invasive fungal infection developed in two of 24 patients who received oral POS and one of 24 patients who recevied oral fluconazole ${ }^{12}$. It should be noted that prophylaxis could lead to selection of resistant strains in patients and environmental flora. It is obvious that more studies focused on primary prophylaxis are needed and primary or secondary antifungal prophylaxis should be evaluated in terms of provided benefits and disadvantages ${ }^{13}$.

Candida species predominated confirmed fungal infections in our study. Colonization of the mucosal membranes by Candida species preceded invasive diseases, while Aspergillus and other molds invade the respiratory tract ${ }^{11}$.

VOR was modified to other antifungal drugs in patients who were infected with Candida species due to antifungal resistance. It should be taken into consideration that breakthrough with Candida infection can develop under VOR treatment due to azole resistance. This was previously shown in Candida species where either AMP-B or CAS should be given as an empirical antifungal drug in accordance with the recommendations of IDSA and MSG/ IFICG/EORTC groups, if there is no evidence to supports an Aspergillus infection $(14,15)$.

\section{Conclusion}

It is obvious that more studies focused on primary prophylaxis are needed and primary or secondary antifungal prophylaxis should be evaluated in terms of provided benefits and disadvantages. Timely and appropriately initiated antifungal treatment is one of the most important factors for a good prognosis for recovery from a neutropenic phase.

\section{Acknowledgements}

This study was approved by local ethics committee. We certify that there is no conflict of interest with any financial organization regarding the subject discussed in the manuscript. We greatly appreciate Prof. Dr. Andreas Voss' recommendations about the manuscript.

\section{References}

1. Freifeld AG, Bow EJ, Sepkowitz KA, Boeckh MJ, Ito JI, Mullen CA, et al. Clinical practice guideline for the use of antimicrobial agents in 
neutropenic patients with cancer: 2010 update by the infectious diseases society of america. Clin Infect Dis 2011; 52: 56-93.

2. de Naurois J, Novitzky-Basso I, Gill MJ, Marti Marti FM, Cullen MH, Roila F. Management of febrile neutropenia: ESMO Clinical Practice Guidelines. Ann Oncol 2010; Suppl 5: 252-256

3. Klastersky J, Paesmans M, Rubenstein EB, Boyer M, Elting L, Feld R, et al. The Multinational Association for Supportive Care in Cancer risk index: A multinational scoring system for identifying low-risk febrile neutropenic Cancer Patients. J Clin Oncol 2000; 18: 3038-3051

4. DeGregorio MW, Lee WM, Linker CA, Jacobs RA, Ries CA. Fungal infections in patients with acute leukemia. Am J Med 1982;73(4):543-8.

5. Walsh TJ, Finberg RW, Arndt C, Hiemenz J, Schwartz C, Bodensteiner D, et al. Liposomal amphotericin B for empirical therapy in patients with persistent fever and neutropenia. National Institute of Allergy and Infectious Diseases Mycoses Study Group. N Engl J Med 1999; 340(10):764-71.

6. Hughes WT, Armstrong D, Bodey GP, Bow EJ, Brown AE, Calandra T, et al. 2002 guidelines for the use of antimicrobial agents in neutropenic patients with cancer. Clin Infect Dis 2002;34(6):730-51

7. Caillot D, Couaillier JF, Bernard A, Casasnovas O, Denning DW, Mannone L, et al. Increasing volume and changing characteristics of invasive pulmonary aspergillosis on sequential thoracic computerised tomography scans in patients with neutropenia. J Clin Oncol 2001; 19(1): 253-9.

8. Maertens J, Theunissen K, Verbeken E, Lagrou $\mathrm{K}$, Verhaegen J, Boogaerts M, et al. Prospective clinical evaluation of lower cut-offs for galactomannan detection in adult neutropenic cancer patients and haematological stem cell transplant recipients. $\mathrm{Br} J$ Haematol 2004;126(6):852-60

9. Cornely OA, Maertens J, Winston DJ, Perfect J, Ullmann AJ, Walsh TJ, et al. Posaconazole vs. fluconazole or itraconazole prophylaxis in patients with neutropenia. $N$ Engl J Med 2007;356(4):348-59.

10. Ullmann AJ, Lipton JH, Vesole DH, Chandrasekar P, Langston A, Tarantolo SR, et al. Posaconazole or fluconazole for prophylaxis in severe graft-versus-host disease. N EnglJ Med. 2007;356(4):335-47.

11. De Pauw BE, Donnelly JP. Prophylaxis and aspergillosis - has the principle been proven? N Engl J Med. 2007;356(4):409-11.

12. Gomes MZR, Lewis RE, Farias PMCM, Wu C, Kontoyiannis DP. Incidence of breakthrough fungal infection during primary antifungal prophylaxis in acute myeloid leukoemia patients in a cancer centre. Clin Microbiol Infect 2012; 18 (Suppl 3); 187

13. El Cheikh J. Secondary Antifungal Prophylaxis in Hematopoietic Stem Cell Transplantation (HSCT)/Acute Leukemia Patients. Curr Infect Dis Rep 2011;13(6):528-35.

14. De Pauw B, Walsh TJ, Donnelly JP, Stevens DA, Edwards JE, Calandra $\mathrm{T}$, et al. Revised Definitions of Invasive Fungal Disease from the European Organization for Research and Treatment of Cancer/Invasive Fungal Infections Cooperative Group and the National Institute of Allergy and Infectious Diseases Mycoses Study Group (EORTC/MSG) Consensus Group. Clin Infect Dis 2008;46(12):1813-21.

15. Iatta R, Caggiano G, Cuna T, Montagna MT. Antifungal susceptibility testing of a 10 -year collection of Candida spp. isolated from patients with candidemia. J Chemother 2011;23(2):92-6. 